\title{
Bradyarrythmias: A Study of Anthropometric, Demographic and Comorbidity Profiles in an African Population
}

\author{
Martins Oluwafemi Thomas ${ }^{1,2^{*}}$, Ezekiel O. Ogunleye ${ }^{1,2}$, Olugbenga O. Olusoji ${ }^{1}$, \\ Augustin Olugbemi ${ }^{1}, \mathbf{O} . \mathrm{O}^{-\mathrm{Ojo}^{1}}$ \\ ${ }^{1}$ Cardiothoracic Surgery Unit, Lagos University Teaching Hospital, Lagos, Nigeria \\ ${ }^{2}$ College of Medicine of University of Lagos-Idiaraba, Lagos, Nigeria \\ Email: *oluwafemithomas@yahoo.com
}

Received September 29, 2013; revised October 29, 2013; accepted November 6, 2013

Copyright (C) 2013 Martins Oluwafemi Thomas et al. This is an open access article distributed under the Creative Commons Attribution License, which permits unrestricted use, distribution, and reproduction in any medium, provided the original work is properly cited.

\begin{abstract}
Background: Bradyarrhythmias are a group of cardiac rhythm disorders that are characterized by bradycardia and they are cosmopolitan in distribution. Their demographic, anthropometric and comorbidity attributes are yet to be clearly established in Africa. Aims and Objectives: This study was conducted to determine the anthropometric, demographic and comorbidity factors in an African population. Methods: We got data from two groups of patients-Group A were bradyarrhythmia patients who already had permanent pacemaker insertion (PPI). Group B were non-cardiac non-debilitated patients of similar age bracket. The sample population consisted of referrals received via clinics, admission through the emergency centres and wards. Their bio-data, hospital identification numbers, ages and gender and other relevant parameters were carefully documented. The ensuing data was analyzed with SPSS 21 statistical software. Results: There were 31 patients in group A (17 male and 14 female patients) and 36 in group B (22 male and 14 female patients). They were all above 20 years of age. Group A had a mean age of $65.8 \pm 4.76$ years while group B had a mean age of $62.2 \pm 4.47$ years. Body Mass Index (BMI $\geq 25 \mathrm{Kg} \cdot \mathrm{m}^{-2}$ ), elevated BP, and diabetes mellitus were important clinical attributes of bradyarrhythmias in the studied group. Discussion and Conclusion: Our study showed that the stated anthropometric, demographic and comorbidity parameters are important attributes for bradyarrhythmias in African population.
\end{abstract}

Keywords: Bradyarrhythmias; Anthropometry; Demography; Comorbidity

\section{Introduction}

Bradyarrhythmias are a group of cardiac rhythm disturbances that are associated with unusually low heart rate. The disorders in this group include heart blocks (all types) and the major fascicular blocks. Bradyarrhythmias can be congenital or acquired. Congenital cases are found in neonates and young children. In this class of patients, prenatal diagnosis improves prognosis because of the possibility of early treatment. A retrospective review of Pediatric Cardiac Care Consortium (PCCC) multi-institutional database was conducted by Shepard and his colleagues [1] to identify premature, low-birth weight neonates who had epicardial pacemaker placement in USA.

${ }^{*}$ Corresponding author.
The study revealed the various challenges associated with management of congenital heart blocks in neonates.

On the other hand the acquired varieties are associated with various aetiological factors like myocardial infarcttion, degeneration of conduction tissue or even in parasitic infestations like Chaga's disease [2]. An unusual occurrence of bradyarrhythmias was found in association with central venous cannulation in a uraemic patient in Taiwan by Huang and his colleagues [3]. In the reported case, there was initial ventricular tachycardia which later gave way to complete heart block.

Ekpe and his colleagues, working in Southeastern Nigeria described symptomatic heart block as a treatable cardiac cause of death which occurs globally and they claimed that permanent artificial cardiac pacing is the 
reliable treatment for it [4]. Pacemakers are life-saving implantable devices that are used to treat various cardiac rhythm disturbances including bradyarrhythmias. Although complete heart block remains a definite indication for permanent pacing, most permanent pacemakers implanted in United States are for Sick Sinus Syndrome [5]. This situation widely contrasts with studies in Africa, where complete heart block is the commonest indication for permanent cardiac pacing $[4,6]$.

A study conducted in United States of America by Block and his colleagues [7] has shown that, for nearly over 40 years, body mass index (BMI) means $(\mu)$ and variation increased in parallel across most baseline weight classifications in USA. From the study, it was noted that individual-level characteristics, especially baseline BMI, were the primary factors in rising BMI.

Epidemiological studies of elderly heart failure patients ( $\geq 85$ years) [8] is characterized by more women, higher systolic blood pressure, lower BMI, and higher incidence of cardiovascular and non-cardiovascular comorbidities. There was more left bundle branch block and increasing risk of ischaemic heart disease. Ankylosing spondylitis, a rheumatologic disorder has been associated with conduction disorders of atria and ventricles alike. This was clearly elucidated in a study by Dik and his colleagues in Netherlands [9]. They concluded that conduction defects are highly prevalent in ankylosing spondylitis, particularly in patients with long-standing disease.

Chest pain is an important symptom in relation to ischaemic events of the heart. In a study conducted by McConaghy and Oza [10] at Ohio State University, it was found that approximately $1 \%$ of primary care office visits are for chest pain and $1.5 \%$ of these patients will have unstable angina or acute myocardial infarction. In view of the fact that myocardial infarction is a common precursor of bradyarrhythmias, chest pain, by extension, can therefore precede some bradyarrhythmias. The significance of this relationship has not been clearly established in literature.

There is a paucity of literature from Africa detailing the various attributes of bradyarrhythmias as discussed in this section of this publication. This formed the basis for this study. It was actually done to identify the anthropometric, demographic and comorbidity profiles of patients who have bradyarrhythmias in an African population. It is expected that this will further enrich the literature and further qualify the relationship of those attributes in an African population.

\section{Methodology}

This is a prospective comparative study of anthropometric parameters,demographic attributes, clinical and co- morbid factors of bradyarrhythmic patients in an African population.

\subsection{Study Design}

We designed a well thought out protocol detailing the various variables that were needed to generate anthropometric, demographic and clinical information about the population to be studied. Two groups of patients were studied for ease of comparison. Group A patients were those who had pacemaker inserted for bradyarrhythmias. Group B patients were non cardiac and non-debilitated patients of similar age range with group A. To generate demographic data, the included variables were patients' bio-data, stating the study identification details like study number, hospital identification number, patients' ages and gender. We also noted the ages at puberty, menarche, parity and menopause as may be applicable to each patient.

The anthropometric variables recorded in the protocols included patients' height and weight.

The clinical variables listed were time of diagnosis of the bradyarrhythmias, presence or absence of chest pain and diabetes. We also noted their blood pressures (systolic and diastolic).

\subsection{Statistical Analysis}

The data for all the measurements were entered into IBM SPSS 21 software for storage and analysis. The derived data were calculated from the raw data. The body mass index (BMI) was calculated using formula BMI $\left(\mathrm{Kg} \cdot \mathrm{m}^{-2}\right)$ $=$ Weight $($ in $\mathrm{Kg}) \times$ Height $(\text { in meters })^{-2}$.

Measure of dispersion was carried out by calculating the frequencies of the variables, the means (at 95\% confidence interval), medians and modes. We also calculated the errors of mean, kurtosis and Skewness.

We tested for normality of the various variables with appropriate formulae and the distributions were tested for Kurtosis and Skewness. Test of statistical significance was done using Chi Square or Students t-test at $\mathrm{P}=0.05$ as may be appropriate. We sought statistical association of diabetes with antihypertensive use in both groups

Analysis of relationship was done by cross-tabulation with IBM SPSS 21 software.

The derived information from above analyses formed the basis for the discussion and conclusions (Vide infra).

\section{Results}

Two groups of subjects were involved in the study. Group A consisted of 31 patients who had bradyarrhythmias and all of them had permanent pacemakers. They were randomly selected at Cardiothoracic Surgical outpatient clinic during follow up. 
Group B were 36 non-cardiac non-debilitated patients that were randomly recruited at the Accident Centre and the wards of the hospital. They were all above 20 years of age. The two groups compared favourably and there was no statistical difference in their distribution.

In all, there were 31 male $(38.9 \%)$ and 36 female $(61.1 \%)$ subjects giving a $\mathrm{M}: \mathrm{F}$ ratio of $1: 1.2$.

\subsection{Demographic Attributes of the Patients}

\subsubsection{Measures of Age Dispersion}

Group A patients had a mean age of $65.8 \pm 4.76$ years $(\mu$ $=65.8 \pm 4.76$ years, $\mathrm{P}=0.05)$. The median age was 68.8 years. It was a normal distribution. The age range was 56 years (Minimum was 28 years and Maximum was 84 years). The standard deviation was 23.28 years. Only one patient $(3.2 \%)$ was less than 40 years. There were 10 patients $(32.3 \%)$ in the $41-60$ year age bracket while 17 patients $(54.8 \%)$ were in the $61-80$ year age bracket. All the patients below age of 50 were females.

Group B subjects had a mean of $62.2 \pm 4.47$ years $(\mu=$ $62.2 \pm 4.47, \mathrm{P}=0.05)$. The median was 64.0 years and the Standard Deviation was 13.673 ( $\delta=13.673$ years). The minimum was 21 years and maximum was 76 years, giving a range of 55 years.

The mean ages of the two groups showed no significance at $30 \mathrm{df}$ using Student " $\mathrm{t}$ " test. The age and gender distribution of Group A patients is expressed in Table 1.

\subsubsection{Gender Attributes}

Groups A and B had a combined sample size of 66 patients. There were 31 males and 36 females giving a combined M:F ratio of $1: 1.2$.

Group A had 17 females and 14 females giving a M:F ratio of 1.2:1. The mean age for attainment of puberty was 13.2 years for both genders. The female subjects had a mean age of 15.1 years for attainment of menarche. The mean age for attainment of menopause was 46.3 years. The average parity was 5.3 among the female subjects in group A. The mean age for males in group A was

Table 1. Showing the age and gender distribution of Group A patients.

\begin{tabular}{cccc}
\hline Age & Male & Female & Total \\
\hline $20-29$ & - & 1 & $1(3.2 \%)$ \\
$30-39$ & - & - & $0(0.0 \%)$ \\
$40-49$ & - & 3 & $3(9.7 \%)$ \\
$50-59$ & 5 & 1 & $6(19.4 \%)$ \\
$60-69$ & 3 & 4 & $7(22.6 \%)$ \\
$70-79$ & 7 & 3 & $10(32.3 \%)$ \\
$80-89$ & 2 & 2 & $4(12.9 \%)$ \\
Total & $\mathbf{1 7}(\mathbf{5 4 . 8 \% )}$ & $\mathbf{1 4} \mathbf{( 4 5 . 2 \% )}$ & $\mathbf{3 1}(\mathbf{1 0 0 . 0 \% )}$ \\
\hline
\end{tabular}

68.2 years and that for the females was 62.9 years. This age difference was statistically significant at $23 \mathrm{df}$ using Chi square test and the likelihood ratio was 28.8 .

Group B had a sample size of 36 subjects. There were $22(61.1 \%)$ female and $14(38.9 \%)$ male patients giving a M:F ratio of 1:1.6. Their mean age for attainment of puberty was 11.7 years and the female patients attained menarche at a mean age of 14.0 years. The mean age for males in Group B is 33.7 years while the mean age for females was 45.1 years. The age and gender distribution for Group B is as expressed in Table 2.

\subsection{Anthropometric Attributes}

The mean height for Group A patients was 1.67 metres. The median was same therefore it was a normal distribution.

The tallest patient was 1.80 metres while the shortest was 1.56 metres.

Details of the height attributes are in Table 3.

The mean weight for the same group was $75.0 \pm 6.31$ $\mathrm{Kg}$.

The mean Body Mass Index (BMI) was $26.2 \mathrm{Kg} \cdot \mathrm{m}^{-2}$. Patients that had BMIs $\leq 25 \mathrm{Kg} \cdot \mathrm{m}^{-2}$ constituted $42.9 \%$ of the cases while $57.1 \%$ had BMIs $\geq 25 \mathrm{Kg} \cdot \mathrm{m}^{-2}$.

Details of anthropometric parameters are in Table 3.

Group B Patients had a mean height of 1.62 meters and it was a normal distribution. The mean weight was 73.3 $\mathrm{Kg}$ and it was also a normal distribution. The BMI, in a like manner had a mean of $28.1 \mathrm{Kg} \cdot \mathrm{m}^{-2}$ and it was a normal distribution. Details of anthropometric parameters for Group B patients are as stated in Table 4.

\subsection{Clinical Attributes}

\subsubsection{Presenting Diagnosis}

There were 31 cases of bradyarrhythmias as described for group A patients. Out of these, 29 (93.5\%) were caused by complete heart block and 2 cases $(6.5 \%)$ were caused by Sick Sinus Syndrome. Group B patients were mainly trauma patients, who otherwise were essential normal.

\subsubsection{Time of Diagnosis to Pacemaker Insertion}

The mean duration of symptoms and/or signs before intervention was 23.6 weeks, the median was 4.5 weeks with a mode of one week. Minimum duration of symptoms and/or signs was one week and the maximum was 144 weeks thereby giving a wide range of 143 weeks. The duration mode of one week was generated by eight patients who had pacemaker insertion within one week of developing the features of bradyarrhythmias.

In all, 14 patients $(45.2 \%)$ had pacemaker implantation within a month of developing clinical features of bradyarrhythmias. It is also important that 10 patients 
Table 2. Showing the age and gender distribution of Group B patients.

\begin{tabular}{cccc}
\hline Age & Male & Female & Total \\
\hline $20-29$ & 6 & 2 & $8(22.2 \%)$ \\
$30-39$ & 4 & 4 & $8(22.2 \%)$ \\
$40-49$ & 2 & 5 & $7(19.4 \%)$ \\
$50-59$ & 2 & 6 & $8(22.2 \%)$ \\
$60-69$ & 1 & 4 & $5(13.9 \%)$ \\
Total & $\mathbf{1 5 ( 4 1 . 7 \% )}$ & $\mathbf{2 1 ( 5 8 . 3 \% )}$ & $\mathbf{3 6}(\mathbf{1 0 0 . 0 \% )}$ \\
\hline
\end{tabular}

Table 3. Showing anthropometric data for Group A patients.

\begin{tabular}{cccc}
\hline Parameters & Weight $(\mathrm{Kg})$ & Height (Meters) & BMI $\left(\mathrm{Kg} \cdot \mathrm{m}^{-2}\right)$ \\
\hline Mean & 75.0 & 1.67 & 26.214 \\
S.E (Mean) & 3.2 & 0.02 & - \\
Median & 72.0 & 1.67 & 25.9 \\
Mode & 52.0 & 1.6 & 20.3 \\
SD & 16.4 & 0.08 & 5.9285 \\
Variance & 269.6 & 0.01 & - \\
Skewness & 0.9 & 0.39 & - \\
SE (Skewness) & 0.5 & 0.58 & - \\
Kurtosis & 0.6 & -0.95 & - \\
SE (Kurtosis) & 0.9 & 1.12 & - \\
Range & 65.0 & 0.24 & 18.5 \\
Minimum & 52.0 & 1.56 & 18.8 \\
Maximum & 117.0 & 1.80 & 37.3 \\
\hline
\end{tabular}

Table 4. Showing anthropometric data for Group B patients.

\begin{tabular}{cccc}
\hline Parameters & Weight $(\mathrm{Kg})$ & Height (Meters) & BMI $\left(\mathrm{Kg} \cdot \mathrm{m}^{-2}\right)$ \\
\hline Mean & 73.3 & 1.62 & 28.1 \\
Median & 73.8 & 1.64 & 27.1 \\
SD & 16.7 & 0.064 & 7.5 \\
Range & 56.0 & 0.21 & 25.3 \\
Minimum & 50.0 & 1.51 & 17.7 \\
Maximum & 106.0 & 1.72 & 43.0 \\
\hline
\end{tabular}

(32.3\%) had pacemaker insertion $\geq 6$ months after developing signs and/or symptoms. Only one patient actually had pacemaker insertion 36 months after onset of the clinical features.

\subsubsection{Blood Pressure Measurements}

The mean systolic blood pressure for group A patients at presentation was $132.3 \mathrm{mmHg}$ and their median was 130 $\mathrm{mmHg}$. The distribution was normal with a standard deviation of 22.6. The maximum systolic BP was 250 while the minimum was $110 \mathrm{mmHg}$ thereby giving a range of
$140 \mathrm{mmHg}$. Taking $120 \mathrm{mmHg}$ as the cutoff point, $74.2 \%$ of the distribution had systolic $\mathrm{BP} \geq 120 \mathrm{mmHg}$. The diastolic BP for Group A had a mean of $80.6 \mathrm{mmHg}$ and a median of $80.0 \mathrm{mmHg}$. The standard deviation was $10.9 \mathrm{mmHg}$. Minimum diastolic BP was $53 \mathrm{mmHg}$ and the maximum was $133 \mathrm{mmHg}$ thereby giving a range of $80 \mathrm{mmHg}$.

The Group B patients had a mean systolic BP of 105 $\mathrm{mmHg}$ and a mode of $110 \mathrm{mmHg}$. The minimum was $110 \mathrm{mmHg}$ and maximum of $203 \mathrm{mmHg}$. The diastolic BP for group B had a mean of $77.6 \mathrm{mmHg}$ and a median of $77.0 \mathrm{mmHg}$. The standard deviation was 13.9. The minimum value was $50 \mathrm{mmHg}$ and the maximum was 133 thereby giving a range of $83 \mathrm{mmHg}$.

Within Group A, 15 out of 31 patients (48.4\%) had significant chest pain before bradyarrhythmias were diagnosed whereas in group B, 2 out of 36 patients $(5.6 \%)$ had significant chest pain before the study.

In group $\mathrm{A}, 65.5 \%$ of the patients were on antihypertensive drugs while $20.0 \%$ of the patients in group B were on antihypertensive drugs.

Diabetes mellitus occurred in 15 out of 31 (48.4\%) of patients in group A while 13 out of 36 patients group B (36.1\%) had diabetes mellitus.

Chi Square test showed significant relationship between diabetes and bradyarrhythmias while the likelyhood ratio was also significant for the studied patients in the group. Same result was generated for diabetes in group B. Pearson's Chi test was applied for analysis of chest pain in group B and there was no significant relationship.

There was significant association of diabetes with antihypertensive drug use among the subjects in groups A and B. In group A, the likelihood ratio was 31.040 at $\mathrm{df}$ of 8 while it was 4.927 at df 2 in group B.

\section{Discussion}

Bradyarrhythmias occur worldwide and no age is exempted. It is cosmopolitan in occurrence, therefore a good knowledge of their attributes will further enrich our understanding of the entity. In spite of these cosmopolitan outlooks of bradyarrhythmias, little is known about occurrence of congenital bradyarrhythmias in Africa. In our studied population, there was no case of congenital bradyarrhythmias in the series. All the cases studied were acquired and they were all adults.

A striking development in this study was noted with reversal of what was thought to be female dominance in distribution of bradyarrhythmias cases. Previous studies (mainly case series) from same location showed overwhelming female preponderance [6,11]. The M:F ratio of $1.2: 1$ is a clear shift from what was found in earlier reports (vide supra) in which M:F ratio ranged from 1:9 to 1:13 from the same location. 
The mean age of patients in the study was $65.8 \pm 4.76$ years $(\mathrm{P}=0.05)$. This is in agreement with a mean age derived from previous studies from Africa. Thomas et al. [6] working in Lagos, southwestern Nigeria got a mean patient age of 62 years for bradyarrhythmic patients.

Gender based age difference was also noted in our study. The male patients had a higher mean age (males 68.2 years, females 62.9 years). The group A subjects (bradyarrhythmic group) attained puberty at a mean age of 13.2 years. This was 1.5 years higher than the mean pubertal age group in the non bradyarrhythmic group.

Further studies are needed to determine the reasons for the difference. Also for unknown reason, all the patients below 50 years in this study were females.

The mean duration from diagnosis to permanent pacemaker implantation (PPI) in our patients was 23.6 weeks. In all, only $25.8 \%$ of them had PPI within one week of diagnosis. This unacceptably prolonged period of waiting is often due to financial reasons in Africa [12]. Although the average running cost of PPI is generally low in Africa compared to the situation in the developed countries, PPI is still largely not affordable in view of the low GDP and inadequate healthcare financing [12]. It is also known that Africans do not accept PPI readily [11]. The referenced study conducted by Thomas et al. in Nigeria established initial reluctance of African patients to accept PPI for treatment of bradyarrhythmias. It was revealed in the study that most African patients accept bradypacing readily only in emergency situations when their choices are limited. This is also a major reason for long duration of waiting before definitive PPI. If we take the mean waiting period from patients' mean age at time of PPI, a rough estimate of patients' age at presentation is still 65.3 years $(0.5$ year less than the age at PPI). This offers no statistically significant advantage to early presenters even though it is better to have PPI as close to the time of diagnosis as possible.

Our sample showed a mean height of 1.67 metres and a mean weight of $75.0 \pm 6.31 \mathrm{Kg}$. Height, as a single parameter had no predictive value for occurrence of bradyarrhythmias. The mean BMI of $26.2 \mathrm{Kg} \cdot \mathrm{m}^{-2}$ showed significant association with occurrence of bradyarrhythmias. It was also significant that more patients had BMI $\geq 25 \mathrm{Kg} \cdot \mathrm{m}^{-2}$. This is an important risk factor identified in the studied population.

Complete heart block continued to be the main type of bradyarrhythmia in this part of the world. It accounted for $93.5 \%$ of the cases studied. In a previous report by Thomas et al. [6], $86.0 \%$ of bradyarrhythmic patients who presented for PPI were complete heart block patients. The implication of this is that, African patients mainly present for PPI because of the severe symptoms often associated with complete heart block. In view of the fact that most patients who had PPI were received through referrals, it means that the referring practitioners also have a role to play in identifying other forms of bradyarrhythmias, for prompt referral for PPI when there are clear indications.

Majority of the studied patients (74.2\%) had systolic blood pressure $\geq 120 \mathrm{mmHg}$. This was statistically significant when compared with the non bradyarrhythmic and non-cardiac group of patients, who had a mean systolic BP of $110.0 \mathrm{mmHg}$.

We believe that BP is an important clinical feature of bradyarrhythmias. Looking at this situation in the context of delayed definitive intervention (vide supra), it means that many patients on the waiting list for PPI would have to manage their elevated BP for the duration before PPI. Attending Physicians therefore, have a duty to balance medications to ensure an adequate and sustained blood flow to the vital organs like the brain and kidneys. To deliberately crash their elevated blood pressures to normal levels in the face of bradyarrhythmias should be avoided.

This study showed a significant association of diabetes mellitus with bradyarrhythmias. We also got an unintended inference of significant association of diabetes mellitus with antihypertensive use in the studied groups (both bradyarrhythmic and non bradyarrhythmic groups). We saw this as being normal in view of the established relationship of diabetes with occurrence of heart diseases. It is therefore our belief that, diabetes mellitus, chest pain, high BMI $\left(\geq 25 \mathrm{Kg} \cdot \mathrm{m}^{-2}\right)$ and elevated BP are major anthropometric and clinical attributes of bradyarrhythmias in this environment. The advantages here are that all the parameters and attributes are largely preventable and manageable and so, by inference, bradyarrhythmias are largely preventable.

\section{Conclusions}

From the foregoing, we came to the realization and conclusion that there is ongoing reversal of female gender dominance of bradyarrhythmias in this environment without any significant change in age distribution of the affected individuals. We also concluded that BMI $\geq 25$ $\mathrm{Kg} \cdot \mathrm{m}^{-2}$, elevated BP, diabetes mellitus and chest pain are important anthropometric and clinical attributes of bradyarrhythmias.

We noted the overwhelming dominance of complete heart block as the main indication for PPI for bradyarrhythmias with the impression that other causes of bradyarrhythmias are not seen as being important indications for PPI. This is a dangerous trend because other neglected indications for PPI in bradyarrhythmias can also cause significant morbidity and mortality.

The significantly long period of time between diagnosis of bradyarrhythmias and PPI also caught our attention. We concluded that this is still an important issue in bra- 
dypacing in Africa.

\section{Recommendations}

In view of the foregoing, we hereby recommend that relevant practitioners of health should promptly identify, refer and encourage bradyarrhythmic patients (all forms) who have genuine indications for PPI at all times.

We also recommend that health administrators and relevant public health departments should take definite steps to prevent and manage the noted preventable attributes of bradyarrhythmias as listed in this report.

\section{REFERENCES}

[1] C. W. Shepard, L. Kochilas, J. M. Vinocur, R. Bryant, B. A. Harvey, S. Bradly, W. Decampli and J. D. Louis, "Surgical Placement of Permanent Epicardial Pacing Systems in Very Low-Birth Weight Premature Neonates. A Review of the Data from the Pediatric Cardiac Care Consortium (PCCC)," World Journal for Pediatric and Congenital Heart Surgery, Vol. 3, No. 4, 2012, pp. 454458. http://dx.doi.org/10.1177/2150135112453178

[2] M. V. Rodriguez, W. Y. Hernandez, A. N. Garcia, C. M. Colato, P. G. Cordoza and L. M. Cardozo, "ELISA seroprevalence of Trypanosomacruzi in a Cohort of Heart Disease Patients," The Journal of Infection in Developing Countries, Vol. 7, No. 4, 2013, pp. 348-354.

[3] Y. C. Huang, J. C. Huang, S. C. Chen, J. M. Chang and H. C. Chen, "Lethal Cardiac Arrhythmias during Central Venous Catheterization in a Uraemic Patient: A Case Report and Review of Literature," Hemodialysis International, Vol. 17, No. 4, 2013, pp. 644-648.

[4] E. E. Ekpe, M. A. Aghaji, S. A. Edaigbini and C. N. Onwuta, "Cardiac Pacemaker Treatment of Complete Heart Block in Enugu a 5-Year Review," Nigerian Medical Journal, Vol. 17, No. 1, 2008, pp. 7-12. http://dx.doi.org/10.4314/njm.v17i1.37346

[5] E. L. James and D. G. Lawrence, "Cardiac Pacemakers and Cardiac Conduction System Abnormalities," In: E. H. Wickland Jr. Ed., Surgery of the Chest, WB Saunders Company, Toronto, 1990, pp. 1597-1634.

[6] M. O. Thomas, D. A. Oke, E. O. Ogunleye and F. A. Adeyanju, "Bradypacing: Indications and Management challenges in Nigeria," $P A C E$, Vol. 30, No. 6, 2007, pp. 761763. http://dx.doi.org/10.1111/j.1540-8159.2007.00747.x

[7] J. P. Block, S. V. Subramanian, N. A. Christakis and A. J. O'Malley, "Population Trends and Variations in Body Mass Index (BMI) from 1971 to 2008 in the Framingham Heart Study Offspring Cohort," PLoS One, Vol. 8, No. 5, 2013, Article ID: e63217. http://dx.doi.org/10.1371/journal.pone.0063217

[8] A. Holmstrom, R. Sigurjonsdottir, M. Edner, A. Jonsson and U. Dahlstrom, "Increased Comorbidities in Heart Failure Patients $\geq 85$ Years but Declined from $>90$ Years: Data from the Swedish Heart Failure Registry," International Journal of Cardiology, Vol. 167, No. 6, 2012, pp. 2747-2752.

[9] V. K. Dik, M. J. Peters, B. A. Dijkmans, I. E. Van der Horst-Bruinsma and M. T. Nurmohamed, "The Relationship between Disease-Related Characters and Conduction Disturbances in Ankylosing Spondylitis," Scandinavian Journal of Rheumatology, Vol. 39, No. 1, 2010, pp. 38-41. http://dx.doi.org/10.3109/03009740903096101

[10] J. R. McConaghy and R. S. Oza, "Outpatient Diagnosis of Acute Chest Pain in Adults," American Family Physician, Vol.87, No. 3, 2013, pp. 177-182.

[11] M. O. Thomas, "Acceptance of Elective Bradypacing by Patients: An African Perspective," PACE, Vol. 35, No. 1, 2012, pp. 58-61. http://dx.doi.org/10.1111/j.1540-8159.2011.03228.x

[12] M. O. Thomas, "Cost Issues in Pacemaker Surgery in Nigeria," Nigerian Quarterly Journal of Hospital Medicine, Vol. 14, No. 2, 2004, pp. 140-142. 